\title{
Programación Separable, Fraccional y Geométrica en Problemas de Optimización
}

\author{
Santiago Cartagena Agudelo \\ Mathematical Engineering \\ Universidad EAFIT \\ scartagenadeafit.edu.co
}

\author{
Camilo Cossio Alzate \\ Mathematical Engineering \\ Universidad EAFIT \\ ccossioadeafit.edu.co
}

\author{
Juan Pablo Ossa Zapata \\ Mathematical Engineering \\ Universidad EAFIT \\ jpossaz@eafit.edu.co
}

May 2021

\section{Abstract}

In the daily development of life, human beings find themselves in the need to make decisions, some more important than others, which will bring future consequences that may or may not be favorable. From this, the need arises to choose the best alternative whenever we are faced with a situation in which we must decide on something, in order to avoid any type of loss or disadvantage. The problem is that generally recognizing the best of the possible is not a trivial task.

Through mathematics, we can find a solution to many of these problems, through their proper modeling and subsequent optimization processes.

In general, such problems are composed of an objective function that must be maximized or minimized subject to a set of constraints. In some cases, reaching the solution is difficult because of the structure of the equations and/or inequalities that describe the objective function or the constraints. Based on the above, the objective of this document is to illustrate specific methods to facilitate the solution of certain problems that meet certain characteristics. The main intention is that the reader understands the mathematical foundation of the methods and learns to apply them. In this order of ideas, the separable programming method, fractional programming, and geometric programming will be discussed. 


\section{Introducción}

En este documento se presentan métodos de programación para resolver problemas de optimización específicos, se presenta teóricamente cada método, se establecen sus condiciones de uso y se dan ejemplos para un mayor entendimiento por parte del lector.

El método de programación separable se utiliza cuando la función objetivo y/o las restricciones son descritas por funciones no lineales, además estas deben poder ser expresadas como la suma de $n$ funciones que dependen de una sola variable. El objetivo principal es aproximar dichas funciones no lineales a través de una función lineal por tramos y así resolver fácimente el problema.

El método de programación fraccional es usada cuando la función objetivo es una razón de dos funciones que, en general, no son lineales. La relación a optimizar a menudo describe algún tipo de eficiencia de un sistema. Entre los principales enfoques que se le dan al problema de programación fraccional está que permiten por ejemplo la identificación de cuellos de botella además de facilitar el proceso de análisis, programación y planeación de la actividad productiva, la medición y evaluación de la eficiencia, y la formulación de estrategias para el mejoramiento de la productividad y rentabilidad de las organizaciones, y es precisamente la importancia que tiene la productividad como indicador de rendimiento en las organizaciones, lo que ha generado que las empresas fijen estrategias para su mejoramiento.

El método de programación geométrica se usa cuando la función objetivo es una función posinomial, las restricciones de desigualdad son posinomiales, y las de igualdad son monomiales. Este tipo de problemas son comunes en lo relacionado a volúmenes, áreas, ubicaciones, entre otros. El objetivo es realizar una transformación mediante cambios de variables para convertir el problema a un problema de optimización convexo.

\section{Desarrollo}

\subsection{Programación separable}

El objetivo principal de este método es reemplazar cada función no lineal por una aproximación lineal a trozos. La programación separable es muy importante ya que permite aproximar un problema no lineal convexo con un modelo de programación lineal que da una precisión arbitraria. Para problemas no convexos la aproximación mediante este método también es válida pero se requiere de un esfuerzo mayor.

Definición 1. Una función $f: X \rightarrow \mathbb{R}, X \subset \mathbb{R}^{n}$ se dice que es separable si $f(x)=\sum_{j=1}^{n} f_{j}\left(x_{j}\right)$, es decir, si $f\left(x_{1}, \ldots, x_{n}\right)$ puede ser expresada como la suma de $n$ funciones de una sola variable $f_{1}\left(x_{1}\right), \ldots, f_{n}\left(x_{n}\right)$. [4]

Algunas funciones no lineales no son separables, pero pueden hacerse separables llevando a cabo las sustituciones apropiadas. Por ejemplo, considere el problema

$$
\min F(x)=e^{x_{1}+x_{2}}
$$

Entonces, sea $f=e^{x_{1}+x_{2}}$, luego $\operatorname{Ln}(f)=x_{1}+x_{2}$ y el problema se convierte en

$$
\min F=f
$$

Sujeto a $\operatorname{Ln}(f)=x_{1}+x_{2}$ 
Y este problema es separable.

Para poder aplicar el método a un problema de programación no lineal, este debe cumplir con que la función objetivo y todas las restricciones son separables.

En un principio se considera una función continua de una sola variable $f(x)$. El intervalo $[a, b]$ para $x$ es particionado en intervalos más pequeños a través de puntos de cuadrícula $a=x_{0}<x_{1}<x 2<\ldots<$ $x_{k}=b$. La función no lineal $f(x)$ es aproximada en el intervalo $\left[x_{l}, x_{l+1}\right]$ de la siguiente forma. Sea $x$ una combinación convexa de $x_{l}$ y $x_{l+1}$, es decir,

$$
x=\lambda x_{l}+(1-\lambda) x_{l+1}
$$

para algún $\lambda \in[0,1]$. Luego

$$
\hat{f}(x)=\lambda f\left(x_{l}\right)+(1-\lambda) f\left(x_{l+1}\right)
$$

La distancia entre cada intervalo puede ser igual o no. Más generalmente, la función $f$ puede ser aproximada en el intervalo $[a, b]$ a través de los puntos $x_{0}, x_{1}, \ldots, x_{k}$ por una función lineal a trozos $\hat{f}$ así:

$$
\begin{aligned}
\hat{f}(x) & =\sum_{l=0}^{k} \lambda_{l} f\left(x_{l}\right) \\
\sum_{l=0}^{k} \lambda_{l} & =1, \quad \lambda_{l} \leq 0, \quad l=0,1, \ldots, k \\
x & =\sum_{l=0}^{k} \lambda_{l} x_{l}
\end{aligned}
$$

donde máximo dos $\lambda_{l}$ son positivos y deben ser adyacentes. En algunos casos $\lambda_{l}$ es llamado peso asociado al punto $x_{l}$.

\subsection{Aproximando el problema separable}

Considere el siguiente problema no lineal separable (PS)

$$
\min f(x)=\sum_{j=1}^{n} f_{j}\left(x_{j}\right)
$$

Sujeto a

$$
g_{i}(x)=\sum_{j=1}^{n} g_{i j}\left(x_{j}\right) \leq b_{j}, i=1, \ldots, m
$$

Teniendo en cuenta que algunas funciones $f_{j}$ y $g_{i j}$ pueden ser lineales, vamos a definir $L$ de la siguiente forma:

$$
L=\left\{j: f_{j} \text { y } g_{i j} \text { son lineales para } i=1, \ldots, m\right\}
$$


Para cada $j \notin L$, considere el intervalo $\left[a_{j}, b_{j}\right], a_{j}, b_{j} \geq 0$. Los puntos $x_{l j}, l=0, \ldots, k_{j}$ de la $j$-ésima variable $x_{j}$ se define como

$$
a_{j}=x_{0 j}<x_{1 j}<\ldots<x_{k_{j} j}=b_{j}
$$

no necesariamente equidistantes. Luego, las funciones $f_{j} \mathrm{y} g_{i j}, i=1, \ldots, m$ se pueden aproximar como

$$
\begin{aligned}
\hat{f}_{j}\left(x_{j}\right) & =\sum_{l=0}^{k_{j}} \lambda_{l j} f_{j}\left(x_{l j}\right), \quad j \notin L \\
\hat{g_{i j}}\left(x_{j}\right) & =\sum_{l=0}^{k_{j}} \lambda_{l j} g_{i j}\left(x_{l j}\right), \quad i=1, \ldots, m ; \quad j \notin L
\end{aligned}
$$

donde

$$
\begin{aligned}
\sum_{l=0}^{k_{j}} \lambda_{l j}=1, \quad j \notin L \\
\lambda_{l j} \geq 0, \quad l=0, \ldots, k_{j} ; \quad j \notin L
\end{aligned}
$$

Si $f_{j}$ y $g_{i j}, i=1, \ldots, m$ son lineales para $j \in L$, su aproximación lineal es trivial

$$
\begin{gathered}
\hat{f}_{j}\left(x_{j}\right)=f_{j}\left(x_{j}\right) \\
\hat{g_{i j}}\left(x_{j}\right)=g_{i j}\left(x_{j}\right) \\
i=1, \ldots, m ; \quad j \in L
\end{gathered}
$$

Ahora, para $k_{j}=1$ tenemos

$$
x_{j}=\lambda x_{0 j}+(1-\lambda) x_{1 j}, \quad \lambda \in[0,1]
$$

además sabemos que $f_{j}\left(x_{j}\right)=c_{j} x_{j}, g_{i j}\left(x_{j}\right)=a_{i j} x_{j}$, como $c_{j}$ y $a_{i j}$ son constantes, entonces

$$
\begin{aligned}
\hat{f}_{j}\left(x_{j}\right) & =\lambda f_{j}\left(x_{0 j}\right)+(1-\lambda) f_{j}\left(x_{1 j}\right) \\
& =\lambda c_{j} x_{0 j}+(1-\lambda) c_{j} x_{1 j} \\
& =c_{j} x_{j} \\
& =f_{j}\left(x_{j}\right)
\end{aligned}
$$

$\mathrm{y}$

$$
\begin{aligned}
\hat{g_{i j}}\left(x_{j}\right) & =\lambda g_{i j}\left(x_{0 j}\right)+(1-\lambda) g_{i j}\left(x_{1 j}\right) \\
& =\lambda a_{i j} x_{0 j}+(1-\lambda) a_{i j} x_{1 j} \\
& =a_{i j} x_{j} \\
& =g_{i j}\left(x_{j}\right)
\end{aligned}
$$


Para cada $i=1, \ldots m$ y $j \in L$

Finalmente la aproximación del problema (PS) sería

$$
\min \hat{f}(x)=\sum_{j \in L} f_{j}\left(x_{j}\right)+\sum_{j \notin L} \hat{f}_{j}\left(x_{j}\right)
$$

Sujeto a

$$
\begin{array}{r}
\hat{g}_{i}(x)=\sum_{j \in L} g_{i j}\left(x_{j}\right)+\sum_{j \notin L} \hat{g_{i j}}\left(x_{j}\right) \leq b_{i}, \quad i=1, \ldots, m \\
x_{j} \geq 0, \quad j=1, \ldots, n
\end{array}
$$

y usando las definiciones de $\hat{f}_{j}$ y $\hat{g_{i j}}$ obtenemos

$$
\left.\min \hat{f}(x)=\sum_{j \in L} f_{j}\left(x_{j}\right)+\sum_{j \notin L} \sum_{l=0}^{k_{j}} \lambda_{l j} f_{j}\left(x_{l j}\right)\right)
$$

Sujeto a

$$
\begin{gathered}
\hat{g}_{i}(x)=\sum_{j \in L} g_{i j}\left(x_{j}\right)+\sum_{j \notin L} \sum_{l=0}^{k_{j}} \lambda_{l j} g_{i j}\left(x_{l j}\right) \leq b_{i}, \quad i=1, \ldots, m \\
\sum_{l=0}^{k_{j}} \lambda_{l j}=1, \quad j \notin L \\
\lambda_{l j} \geq 0, \quad l=0, \ldots, k_{j} ; \quad j \notin L \\
x_{j} \geq 0, \quad j \in L
\end{gathered}
$$

Las variables para este problema son $x_{j}, j \in L \mathrm{y} \lambda_{l j}, l=0, \ldots, k_{j}, j \notin L$. La función objetivo y las restricciones son funciones lineales por tramos. Máximo dos $\lambda_{l j}$ adyacentes son positivos para $j \notin L$

La precisión de la solución óptima depende del tamaño de los intervalos, mientras más pequeños sean, mejore será la aproximación.

Es importante resaltar que la programación separable garantiza una aproximación del óptimo global del problema original solamente cuando se minimiza una función convexa o se maximiza una función cóncava en un conjunto convexo de restricciones.

Además, si las condiciones que definen un programa convexo no se satisfacen, puede ocurrir lo siguiente [2]:

- Se encuentra una aproximación a un óptimo global.

- Se encuentra una aproximación a un óptimo local.

- La solución del problema aproximado puede ser infactible respecto al problema original. 


\subsection{Ejemplo}

Resolver el siguiente problema:

$$
\text { Minimizar } f(x)=x_{1}^{2}-6 x_{1}-x_{2}+9
$$

Sujeto a

$$
\begin{gathered}
g_{1}(x)=2 x_{1}+3 x_{2} \leq 24 \\
g_{2}(x)=x_{1}-2+2 x_{2} \leq 15 \\
g_{3}(x)=3 x_{1}+2 x_{2} \leq 24 \\
x_{1} \geq 0 \text { y } 0 \leq x_{2} \leq 4
\end{gathered}
$$

En primer lugar, se decide graficar la región factible del problema.

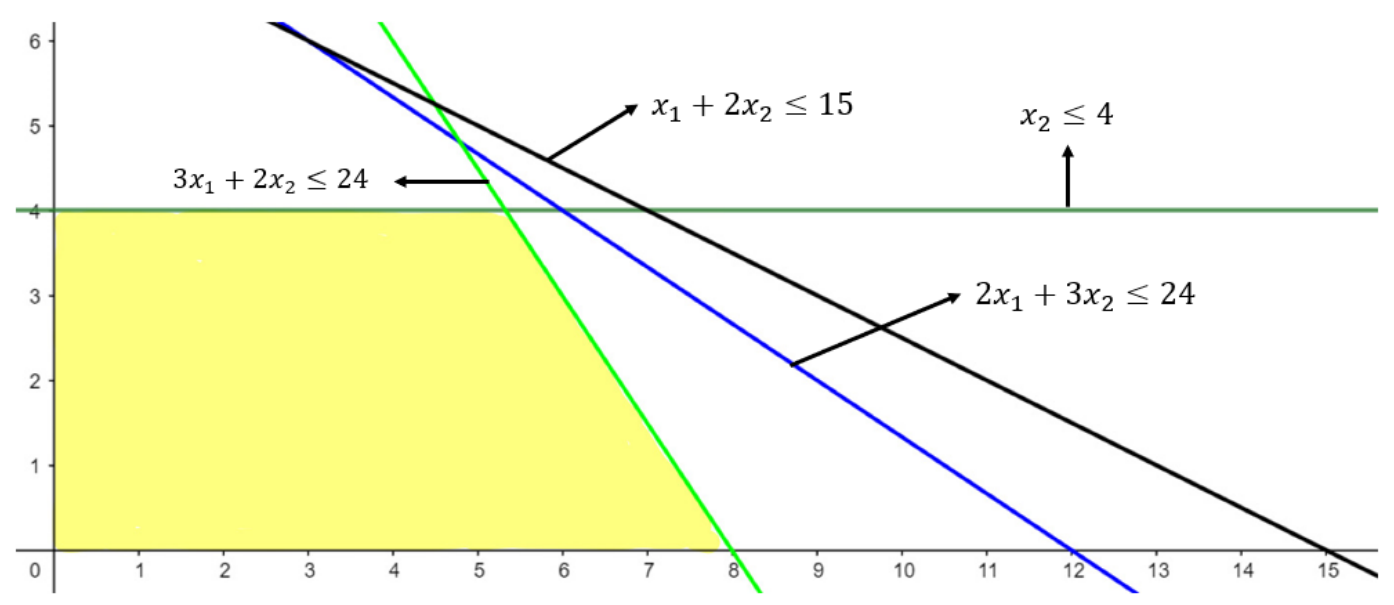

Figura 1: Región factible

Como podemos ver, la región es convexa y por tanto un mínimo global de $f$ en la región puede ser encontrado usando la aproximación lineal.

El siguiente paso es separar la función objetivo y las restricciones como se muestra a continuación

\begin{tabular}{|c|c|c|}
\hline $\mathbf{j}$ & 1 & 2 \\
\hline$f_{j}\left(x_{j}\right)$ & $x_{1}^{2}-6 x_{1}+9$ & $-x_{2}$ \\
$g_{1 j}\left(x_{j}\right)$ & $2 x_{1}$ & $3 x_{2}$ \\
$g_{2 j}\left(x_{j}\right)$ & $x_{1}$ & $2 x_{2}$ \\
$g_{3 j}\left(x_{j}\right)$ & $3 x_{1}$ & $2 x_{2}$ \\
\hline
\end{tabular}

Cuadro 1: Separación de variables

Como podemos ver, solamente la función $f_{1}\left(x_{1}\right.$ es no lineal, por esto basta con aproximar la misma. Para este caso $L=\{2\}$ porque $f_{2}\left(x_{2}\right)$ y $g_{i 2}, i=1,2,3,4$ son lineales. En la figura 1 podemos ver que $x_{1} \leq 8$ cuando $x_{2}=0$, por lo que concluimos que $x_{1} \in[0,8]$ y podemos partir dicho intervalo así:

$$
x_{l 1}=l, l=0,1, \ldots, 8
$$


Los valores de $f_{1}\left(x_{1}\right)$ se muestran en el siguiente cuadro

\begin{tabular}{|c|ccccccccc|}
\hline$x_{l 1}$ & 0 & 1 & 2 & 3 & 4 & 5 & 6 & 7 & 8 \\
\hline$f_{1}\left(x_{l 1}\right)$ & 9 & 4 & 1 & 0 & 1 & 4 & 9 & 16 & 25 \\
\hline
\end{tabular}

Cuadro 2: Valores de $f_{1}\left(x_{1}\right)$

A partir de lo anterior obtenemos

$$
\begin{aligned}
\hat{f}_{1}\left(x_{1}\right) & =9 \lambda_{01}+4 \lambda_{11}+1 \lambda_{21}+0 \lambda_{31}+1 \lambda_{41}+4 \lambda_{51}+9 \lambda_{61}+16 \lambda_{71}+25 \lambda_{81} \\
x_{1} & =0 \lambda_{01}+1 \lambda_{11}+2 \lambda_{21}+3 \lambda_{31}+4 \lambda_{41}+5 \lambda_{51}+6 \lambda_{61}+7 \lambda_{71}+8 \lambda_{81}
\end{aligned}
$$

Reemplazamos las ecuaciones anteriores en la función objetivo y restricciones, el problema queda así

$$
\operatorname{Minimizar} \hat{f}=9 \lambda_{01}+4 \lambda_{11}+\lambda_{21}+\lambda_{41}+4 \lambda_{51}+9 \lambda_{61}+16 \lambda_{71}+25 \lambda_{81}-x_{2}
$$

Sujeto a

$$
\begin{aligned}
& 2 \lambda_{11}+4 \lambda_{21}+6 \lambda_{31}+8 \lambda_{41}+10 \lambda_{51}+12 \lambda_{61}+14 \lambda_{71}+16 \lambda_{81}+3 x_{2} \leq 24 \\
& \lambda_{11}+2 \lambda_{21}+3 \lambda_{31}+4 \lambda_{41}+5 \lambda_{51}+6 \lambda_{61}+7 \lambda_{71}+8 \lambda_{81}+2 x_{2} \leq 15 \\
& 3 \lambda_{11}+6 \lambda_{21}+9 \lambda_{31}+12 \lambda_{41}+15 \lambda_{51}+18 \lambda_{61}+21 \lambda_{71}+24 \lambda_{81}+2 x_{2} \leq 24 \\
& \lambda_{01}+\lambda_{11}+\lambda_{21}+\lambda_{31}+\lambda_{41}+\lambda_{51}+\lambda_{61}+\lambda_{71}+\lambda_{81}=1 \\
& x_{2} \geq 0, \quad \lambda_{l 1} \geq 0, \quad l=0,1, \ldots, 8
\end{aligned}
$$

Procedemos a solucionar el problema con ayuda del software MatLab

$\%$ Solucion ejemplo

$\%$ Funcion objetivo

$\mathrm{f}=\left[\begin{array}{llllllllll}9 & 4 & 1 & 0 & 1 & 4 & 9 & 16 & 25 & -1\end{array}\right]$

$\%$ Restricciones de desigualdad

$\mathrm{A}=\left[\begin{array}{llllllllll}0 & 2 & 4 & 6 & 8 & 10 & 12 & 14 & 16 & 3 \text {; }\end{array}\right.$

$\begin{array}{llllllllll}0 & 1 & 2 & 3 & 4 & 5 & 6 & 7 & 8 & 2 ;\end{array}$

$\begin{array}{lllllllllll}0 & 3 & 6 & 9 & 12 & 15 & 18 & 21 & 24 & 2 \text {; }\end{array}$

$\left.0 \begin{array}{llllllllll}0 & 0 & 0 & 0 & 0 & 0 & 0 & 0 & 0 & 1]\end{array}\right]$

$\mathrm{b}=[24 ; 15 ; 24 ; 4]$

$\%$ Restricciones de igualdad

Aeq $=\left[\begin{array}{llllllllll}1 & 1 & 1 & 1 & 1 & 1 & 1 & 1 & 1 & 0\end{array}\right]$

beq $=[1]$

$\%$ Limite inferior

$1 \mathrm{~b}=\operatorname{zeros}(1,10)$

$\%$ Solucion

$\left[\begin{array}{ll}\mathrm{x} & \mathrm{fval}\end{array}\right]=\operatorname{linprog}(\mathrm{f}, \mathrm{A}, \mathrm{b}$, Aeq, beqlb $)$

La solución fue 


$$
\begin{aligned}
\lambda_{01} & =0 \\
\lambda_{11} & =0 \\
\lambda_{21} & =0 \\
\lambda_{31} & =1 \\
\lambda_{41} & =0 \\
\lambda_{51} & =0 \\
\lambda_{61} & =0 \\
\lambda_{71} & =0 \\
\lambda_{81} & =0 \\
x_{2} & =4 \\
f & =-4
\end{aligned}
$$

y sabemos que

$$
\begin{aligned}
x_{1} & =0 \lambda_{01}+1 \lambda_{11}+2 \lambda_{21}+3 \lambda_{31}+4 \lambda_{41}+5 \lambda_{51}+6 \lambda_{61}+7 \lambda_{71}+8 \lambda_{81} \\
& =0 * 0+1 * 0+2 * 0+3 * 1+4 * 0+5 * 0+6 * 0+7 * 0+8 * 0 \\
& =3
\end{aligned}
$$

En conclusión, la solución óptima del problema original es

$$
\begin{aligned}
& x_{1}=3 \\
& x_{2}=4
\end{aligned}
$$

y el valor óptimo es

$$
f=-4
$$

\subsection{Programación fraccional}

En esta sección se aborda el tema de la programación fraccional, el cual junto con la programación separable y la programación geométrica es otro de los problemas de programación No lineal. Para aterrizar el concepto de programación fraccional, es posible partir de que la función objetivo se encuentre en forma de fracción, esto es matemáticamente, la razón de dos funciones. La programación fraccionada se ocupa de la optimización de una o varias proporciones de valores reales extendidos, es decir, que la programación fraccional consiste en optimizar el cociente de dos funciones que por lo general son No lineales sujeto a un conjunto de restricciones. 


\subsubsection{Programa fraccional(forma estándar)}

$$
\operatorname{Minimizar} \frac{p^{T} * x+\alpha}{q^{T} * x+\beta}
$$

Sujeto a:

$$
\begin{gathered}
A * x \leq b \\
x \geq 0
\end{gathered}
$$

donde $\mathbf{p}$ y $\mathbf{q}$ son vectores fila de dimensión $\mathrm{n}, \mathbf{b}$ es un vector de dimensión $\mathrm{m}, \mathbf{A}$ es una matriz de dimensión $\mathrm{m} x \mathrm{n}, \mathrm{y} \alpha, \beta$ son escalares. Es importante decir, que este problema puede transformarse en un Problema de Programación Lineal mediante el teorema de Charnes y Cooper que será elemento de análisis más adelante.

Una distinción clásica en la teoría de la programación fraccional, es diferenciar entre problemas fraccionales de simple razón y de múltiples razones.

Definición 2(Programación fraccional de simple relación). Para empezar con problemas de programación fraccional de simple relación, sea $\mathbf{B} \subset \mathbb{R}^{n}$ un conjunto cerrado y no vacío y $\mathrm{g}, \mathrm{h}: \mathbb{R}^{n} \rightarrow[-\infty,+\infty]$ funciones de valor real extendidas que son de valor finito en $\mathrm{B}$. Asumiendo que $\mathrm{h}(\mathrm{x})>0 \forall \mathrm{x} \in B$, consideremos el problema No lineal:

$$
P(1): \min _{x \in B} \frac{g(x)}{h(x)}
$$

El problema (P1) se denomina programa fraccionario de razón única. En la mayoría de las aplicaciones, la región factible no vacía tiene más estructura y está dada por:

$$
B=\left\{x \in C: h_{k}(x) \leq 0, k=1, \ldots, l\right\}
$$

Con $\mathbf{C} \subset \mathbb{R}^{n}$ y $h_{k}: \mathbb{R}^{n} \rightarrow \mathbb{R}, 1 \leq k \leq l$ algún conjunto de funciones continuas de valor real. Hasta ahora, las funciones en el numerador y denominador no se especificaron. Si $f, g$ y $h_{k}: \mathbb{R}^{n} \rightarrow \mathbb{R}, 1 \leq k \leq l$ son funciones afines y $C=\mathbb{R}_{+}^{n}$ denota los reales no negativos, luego el problema de optimización $\mathrm{P}(1)$ es conocido como problema de programación fraccional lineal de una sola razón. Además, se llama al problema $\mathrm{P}(1)$ programa fraccionario cuadrático de una sola relación si $C=\mathbb{R}_{+}^{n}$, las funciones f y g son cuadráticas y la función $h_{k}: \mathbb{R}^{n} \rightarrow \mathbb{R}, 1 \leq k \leq l$ es afín. El problema de minimización $\mathrm{P}(1)$ es llamado programa fraccional convexo de una sola relación si $C$ es un conjunto convexo, $h_{k}: \mathbb{R}^{n} \rightarrow \mathbb{R}, 1 \leq k \leq l$ y f son funciones convexas y g es una función cóncava positiva en B.Además, se supone que f no es negativo en B si g no es afín. En caso de un problema de maximización, el programa fraccionario de razón única se denomina programa fraccionario cóncavo de razón simple si f es cóncava y g es convexa. Bajo estos supuestos de convexidad/concavidad restrictivas el problema de minimización (P1) es en general un problema no convexo. En algunas aplicaciones, aparece más de una razón en la función objetivo, si más de una fracción aparece en el objetivo, el problema es llamado problema fraccional multi-razón. 
Definición 3(Programación fraccional de más de una relación). En cuanto a los problemas de programación fraccional de más de una relación tenemos dos casos principalmente [3], los cuales son:

- Programación fraccionada por suma de razones:

$$
\sum_{i=1}^{m} \frac{g_{i}(x)}{h_{i}(x)} \rightarrow \text { Min }, x \in S
$$

Con $h_{i}(x)>0, \forall i=1: m$ y $x \in S$

- Programación fraccionada multiobjetivo

$$
\left(\frac{g_{1}(x)}{h_{1}(x)}, \ldots, \frac{g_{m}(x)}{h_{m}(x)}\right) \rightarrow \operatorname{Min}, x \in S
$$

Con $h_{i}(x)>0, \forall i=1: m$ y $x \in S$

Por otra parte, la programación fraccional presenta múltiples aplicaciones en problemas de la vida real bien sea de tipo maximizar o de tipo minimizar, algunos de ellos son Minimización de la relación costotiempo, maximización de la relación producto-insumo, maximización del beneficio-capital, La relación entre beneficios e ingresos, maximización de la eficiencia energética en redes inalámbricas, o se maximiza la razón de la producción entre las horas-hombre empleadas que es conocids como productividad, entre otros, de allí la importancia de conocer a fondo esta variante de la programación No lineal.

Teorema 1. (Programación fraccional). Si el conjunto factible

$$
X=\{x \mid A x \leq b \quad y \quad x \geq 0\}
$$

Es acotado y no vacío, y si $q^{T} x+\beta>0$ para cada $x \in X$, la ecuación (1) y (2) equivale al PPL:

$$
\text { Minimizar } p^{T} y+\alpha z
$$

Sujeto a

$$
\begin{aligned}
A y-b z & \leq 0 \\
q^{T} y+\beta z & =1 \\
y & \geq 0 \\
x & \geq 0
\end{aligned}
$$

Este teorema se basa en la siguiente transformación:

$$
z=\frac{1}{q^{T} x+\beta} \text { y } y=z x
$$




\subsection{Ejemplo}

Supóngase

$$
\text { Minimizar } z=\frac{x_{1}+1}{x_{2}+2}
$$

s.a

$$
\begin{gathered}
x_{1}+x_{2} \leq 1 \\
x_{1}, x_{2} \geq 0
\end{gathered}
$$

Para transformar este problema de programación lineal fraccional en uno lineal, se realiza la transformación

$$
z=\frac{1}{x_{2}+1} ; y=z x
$$

Y obtenemos:

$$
\text { Minimizar } y_{1}+z
$$

Sujeto a

$$
\begin{aligned}
y_{1}+y_{2}-z & \leq 0 \\
y_{2}+2 z & =1 \\
y_{1}, y_{2}, z & \geq 0
\end{aligned}
$$

\subsection{Ejemplo}

Supóngase el siguiente problema de programación lineal fraccional.

$$
\text { Minimizar } z=\frac{x_{1}+1}{x_{2}+2}
$$

Sujeto a

$$
\begin{gathered}
x_{1}+x_{2} \leq 1 \\
x_{1}, x_{2} \geq 0
\end{gathered}
$$

Para transformar este problema de programación lineal fraccional en uno lineal, se realiza la transformación

$$
z=\frac{1}{x_{2}+1} ; y=z x
$$

Y se obtiene el PPL

$$
\text { Minimizar } y_{1}+z
$$

Sujeto a

$$
\begin{aligned}
y_{1}+y_{2}-z & \leq 0 \\
y_{2}+2 z & =1 \\
y_{1}, y_{2}, z & \geq 0
\end{aligned}
$$




\subsection{Programación geométrica}

Para comprender el concepto de la programación geométrica, es importante conocer las definiciones de función monomial y posinomial:

\subsubsection{Funciones monomiales}

Sean $x_{1}, \ldots, x_{n}$ variables reales positivas, y sea $x=\left(x_{1}, \ldots, x_{n}\right)$ un vector con los componentes $x_{i}$. Una función de la forma

$$
g(x)=c x_{1}^{a_{1}} x_{2}^{a_{2}} \ldots x_{n}^{a_{n}}
$$

donde $c>0$ y $a_{i} \in \mathbf{R}$ se conoce como una función monomial, en el contexto de la programación geométrica.

Las funciones monomiales son cerradas bajo multiplicación y división, y una función monomial elevada a una potencia también es una función monomial.

\subsubsection{Funciones posinomiales}

Si $g_{1}(x), \ldots, g_{K}(x)$ son funciones monomiales, entonces una función de la forma

$$
f(x)=\sum_{k=1}^{K} g_{k}(x)
$$

se conoce como una función posinomial. Estas funciones son cerradas bajo operaciones de suma, multiplicación, y escalado no negativo.

\subsubsection{Programa geométrico}

Un programa geométrico[1] es un problema de optimización de la forma

$$
\text { minimizar } f_{0}(x)
$$

Sujeto a:

- $f_{i}(x) \leq 1, i=1, \ldots, m$

- $g_{i}(x)=1, i=1, \ldots, p$

- $x_{i}>0, \forall i$

Donde $f_{i}$ son funciones posinomiales, $g_{i}$ son monomiales, y $x_{i}$ son las variables de decisión.

\subsection{Ejemplo}

Supóngase que queremos optimizar el volúmen de una caja con altura $h$, ancho $w$ y profundidad $d$ que estará colocada contra una pared de área $A_{\text {wall }}$ y sobre un puso con área $A_{f l r}$. Además, la caja debe tener una relación de aspecto dada por un intervalo particular. Esto nos daría un problema inicial de la forma:

\section{Maximizar $h w d$}

Sujeto a: 
- $2(h w+h d) \leq A_{\text {wall }}$

- $w d \leq A_{f l r}$

- $\alpha \leq h / w \leq \beta$

- $\gamma \leq d / w \leq \delta$

Que no es un problema de optimización geométrica, pero puede ser convertido en uno utilizando las transformaciones algebraicas adecuadas:

Minimizar $h^{-1} w^{-1} d^{-1}$

Sujeto a:

- $\left(2 / A_{\text {wall }}\right) h w+\left(2 / A_{\text {wall }}\right) h d \leq 1$

- $\left(1 / A_{f l r}\right) w d \leq 1$

- $a h^{-1} w \leq 1$

- $(1 / \beta) h w^{-1} \leq 1$

- $\gamma w d^{-1} \leq 1$

- $(1 / \delta) w^{-1} d \leq 1$

\section{Estado del arte}

\subsection{Programación separable}

Debido a su amplio rango de aplicación, el algoritmo de programación separable ha sido usado en muchos campos, este puede ser usado para resolver cualquier tipo de problema de optimización que cumpla con las condiciones ya mencionadas en el desarrollo del documento y facilita en gran medida el trabajo, además requiere menos capacidad computacional de las máquinas ya que se trataría como un problema lineal. A través de los años, el método no ha sido sometido a modificaciones significativas. El proceso, resumidamente es:

- Separar las funciones

- Establecer la cantidad de intervalos y los puntos de ruptura (no necesariamente equidistantes), resaltando que entre más intervalos mejor es la aproximación pero, de la misma forma, aumenta el tamaño del problema ya que a mayor cantidad de intervalos, mayor cantidad de variables, por lo que hay que alcanzar un equilibrio.

- Reemplazar los valores de las nuevas variables en la función objetivo y las restricciones.

- Formular el nuevo problema de programación lineal

- Resolver el problema.

- Reemplazar las soluciones encontradas en las variables originales.

Y así llegamos a la solución óptima de un problema no lineal, que mediante las transformaciones correctas, se convirtió en uno lineal. 


\subsection{Programación fraccional}

En cuanto a lo que programación fraccional se refiere, sus métodos de solución en general han sufrido cambios poco significativos, pues hoy en día se siguen usando métodos del siglo $X X$ para su solución como el dado por el teorema de Charnes y Cooper mencionado en el apartado 3.4, el cual data del año 1962. También, el favorito por los estudiantes y el más famoso a lo largo de estos años según [3] es el método convexo-simplex desarrollado por Zangwill en el año 1967, el cual se utiliza para resolver el valor óptimo de la función objetivo convexa sujeto a restricciones de desigualdad lineal. Además, otro de los famosos métodos que se siguen manteniendo para aplicar la programación fraccional a la solución de problemas de la vida real es el procedimiento de Gilmore y Gomory que viene del año 1963.

\subsection{Programación geométrica}

Los métodos de solución de programación geométrica no han cambiado mucho, debido a la transformación fundamental que los hace importantes para empezar.

La solución de problemas de programación geométrica se centra alrededor del hecho de que es posible convertir todos los problemas de programación geométrica a problemas no lineares pero convexos con el siguiente procedimiento[1]:

- Cada variable de decisión $x_{i}$ se transforma a una $y_{i}=\log x_{i}$, de forma que $x_{i}=e^{y_{i}}$.

- La función objetivo se convierte en minimizar $\log f_{0}$.

- Las inecuaciones $f_{i} \leq 1$ se convierten en $\log f_{i} \leq 0$

- Las ecuaciones $g_{i}=1$ se convierten en $\log g_{i}=0$

De esta forma las igualdades $g$ adquieren la forma:

$$
\log g\left(e^{y}\right)=\log c+a_{1} \log x_{1}+\ldots+a_{n} \log x_{n}=\log c+a_{1} y_{1}+\ldots+a_{n} y_{n}
$$

Que son afines, y se pueden transformar a:

$$
a_{1} y_{1}+\ldots a_{n} y_{n}=-\log c
$$

Que es lineal.

Similarmente, es posible demostrar que $\log f\left(e^{y}\right)$ será una función convexa cuando $f$ es una función posinomial.

De esta forma el problema se convierte en no-lineal pero convexo, y puede ser resuelto por cualquier método de solución de problemas convexos. Por este motivo no ha habido mucho desarrollo en cuanto a los métodos de solución de problemas de programación geométrica, sino que el desarrollo es más centrado a encontrar mejores formas de resolver problemas de optimización convexos, y a encontrar formas de convertir problemas de programación geométrica particulares a unos que tengan restricciones incluso más sencillas de resolver. 


\section{Conclusiones}

Existen varias clases de problemas que en primera instancia se presentan como no lineales y por lo tanto difíciles de resolver. Sin embargo, si cumplen las condiciones adecuadas, estos problemas pueden ser transformados de manera algebraica en problemas más sencillos para los cuales ya existen métodos definidos. Esto permite resolver problemas mucho más grandes, lo que puede ser de gran importancia en las industrias y en el desarrollo científico.

Este estudio supo identificar de manera oportuna cada uno de estos métodos y su aplicación en el mundo real, ahondando en la importancia de los mismos y tomando ideas bastante interesantes que las hace importantes a la hora de llevar a cabo procesos de optimización. Por otra parte, genera curiosidad el hecho que en la mayoría de los casos, los métodos de solución a los diferentes problemas analizados en el trabajo han sufrido pocos cambios significativos, lo cual ciertamente ha plasmado ideas para abordar los métodos mencionados anteriormente en asignaturas posteriores en el proceso académico, que pueden ser materia de investigación.

Minimizar $-h^{\prime}-w^{\prime}-d^{\prime}$

Sujeto a:

- $\log \left(\frac{2}{A_{\text {wall }}} e^{h^{\prime} w^{\prime}}+\frac{2}{A_{\text {wall }}} e^{h^{\prime} d^{\prime}}\right) \leq 0$

- $\log \left(\frac{1}{A_{f l r}}\right)+w^{\prime}+d^{\prime} \leq 0$

- $\log (\alpha)-h^{\prime}+w^{\prime} \leq 0$

- $\log \left(\frac{1}{\beta}\right)+h^{\prime}-w^{\prime} \leq 0$

- $\log (\gamma)+w^{\prime}-d^{\prime} \leq 0$

- $\log \left(\frac{1}{\delta}\right)-w^{\prime}+d^{\prime} \leq 0$

\section{Referencias}

[1] Stephen Boyd y col. "A Tutorial on Geometric Programming”. En: Optimization and Engineering 8 (mayo de 2007), págs. 67-127. DOI: 10.1007 /s11081-007-9001-7.

[2] Paul A. Jensen y Jonathan F. Bard. "Nonlinear Programming Methods.S1 Separable Programming". En: Operations Research Models and Methods (), págs. 1-10.

[3] Nigussie Goshu N. "Fractional programming". En: Addis Ababa Science and Technology (2014).

[4] Stefan M. Stefanov. Applied optimization, Separable Programming. Springer-Science+Business Media, B.Y., 2001. 


\section{Estabilidad}

Con el objetivo de encontrar la condición de estabilidad para la ecuación de calor con un espaciado de malla igual, el método de estabilidad de Von-Neumann es definido como sigue:

$$
\begin{gathered}
\frac{U_{1 j}^{n+1}-U_{i j}^{n}}{\Delta t}=\frac{U_{i+1, j}^{n}-2 U_{i, j}^{n}+U_{i-1, j}^{n}}{(\Delta x)^{2}}+\frac{U_{i+1, j}^{n}-2 U_{i, j}^{n}+U_{i-1, j}^{n}}{(\Delta y)^{2}} \\
u(x, y, t)=e^{i(k, l)(x, y)}
\end{gathered}
$$

Ahora, expandiendo la fomula exponencial de Euler obtenemos:

$$
\frac{G-1}{\Delta t}=\frac{e^{i k \Delta x}-2+e^{i k \Delta x}}{(\Delta x)^{2}}+\frac{e^{i l \Delta x}-2+e^{i l \Delta y}}{(\Delta y)^{2}}
$$

Lo cual puede reescribirse como:

$$
\rightarrow G=1-2 \frac{\Delta t}{(\Delta x)^{2}}(1-\cos (k \Delta x))-2 \frac{\Delta t}{(\Delta y)^{2}}(1-\cos (l \Delta y))
$$

Considerando el peor de los casos tenemos:

$$
k \Delta x=\pi=l \Delta y \rightarrow G=1-4 \frac{\Delta t}{(\Delta x)^{2}}-4 \frac{\Delta t}{(\Delta y)^{2}}
$$

Finalmente la condición de estabilidad será:

$$
\frac{\Delta t}{(\Delta x)^{2}}+\frac{\Delta t}{(\Delta y)^{2}} \leq \frac{1}{2}=\Delta t \leq \frac{1}{2}\left((\Delta x)^{2}+(\Delta y)^{2}\right)
$$

Además si $\Delta x=\Delta y=h$ :

$$
\frac{h^{2}}{4}
$$

